\title{
"Omics" Approaches for Sleep and Circadian Rhythm Research: Biomarkers for Identifying Differential Vulnerability to Sleep Loss
}

\author{
Namni Goel
}

Published online: 11 January 2015

(C) Springer International Publishing AG 2015

\begin{abstract}
The two-process model of sleep-wake regulation asserts a neurobiological drive for sleep that varies homeostatically (increasing as a saturating exponential during wakefulness and decreasing in a similar manner during sleep) and a circadian process that neurobiologically modulates the homeostatic drive for sleep and waking performance and alertness. Sleep deprivation increases homeostatic sleep drive and degrades waking neurobehavioral functions as reflected in fatigue, sleepiness, attention, memory, and cognitive speed. Notably, there are robust individual differences in neurobehavioral responses to sleep loss which are trait-like and phenotypic and not explained by baseline functioning or other possible predictors. This review discusses "omics" methodologies (transcriptomics, epigenomics, and metabolomics) in sleep and circadian rhythm research. Since the molecular mechanisms underlying differential vulnerability remain virtually unknown, such methodologies can be used to yield biomarkers for predicting individual differences in neurobehavioral responses to sleep loss in humans. Reliable prediction of who is more or less likely to experience neurobehavioral decrements from sleep loss would provide more targeted use of biological countermeasures and optimization of personnel in a variety of occupational settings.
\end{abstract}

Keywords Biomarkers $\cdot$ Epigenomics $\cdot$ Metabolomics · Transcriptomics $\cdot$ Sleep deprivation $\cdot$ Performance $\cdot$ Circadian rhythms $\cdot$ Individual differences

This article is part of the Topical Collection on Function of Sleep

N. Goel $(\bowtie)$

Division of Sleep and Chronobiology, Department of Psychiatry, Unit for Experimental Psychiatry, University of Pennsylvania

Perelman School of Medicine, 1017 Blockley Hall, 423 Guardian

Drive, Philadelphia, PA 19104-6021, USA

e-mail: goel@mail.med.upenn.edu

\section{Introduction}

Sleep and wakefulness are modulated by an endogenous biological clock in the suprachiasmatic nuclei of the anterior hypothalamus. The biological clock drives waketime and bedtime and also modulates waking behavior, as observed in cognitive performance and sleepiness, generating circadian rhythmicity in neurobehavioral measures [1, 2]. Conceptualizations of the temporal modulation of sleep and wakefulness and of waking cognitive functions have been represented in the two-process model of sleep regulation and its variants [3-5]. This sleep regulation model has been applied to the temporal profiles of sleep $[4,6]$ and wakefulness [7]. The model consists of a sleep homeostatic process (S) and a circadian process $(\mathrm{C})$ that interact to determine sleep onset and offset timing and waking neurobehavioral functioning stability $[1,2,8]$. Process $\mathrm{S}$ represents the drive for sleep that increases during wakefulness (observed when wakefulness is maintained beyond habitual bedtime into the night and subsequent day) and decreases during sleep (representing recuperation via sleep). Sleep is triggered when this homeostatic drive increases above a particular threshold, and wakefulness is invoked when the drive decreases below a different particular threshold. Process $\mathrm{C}$ represents daily oscillatory modulation of such threshold levels. The circadian system may actively promote wakefulness more than sleep [9]. Even after a night without sleep, the circadian drive for wakefulness can be experienced as spontaneously enhanced alertness in the early evening; however, sleep loss can increase homeostatic pressure so markedly that waking cognitive functions are degraded even at this peak of the circadian drive for wakefulness [10].

Sleep disorders, medical conditions, stress or emotional distress, work, and social or domestic responsibilities all can produce sleep loss [11]. For most individuals, sleep loss causes substantial risks by increasing fatigue and sleep 
propensity and by producing deficits in neurobehavioral functions including cognitive speed and working memory, vigilant attention, and executive functions [11-13].

Notably, subjects exposed to total sleep deprivation or chronic sleep restriction show differential vulnerability to sleep loss, demonstrating phenotypic, trait-like individual differences in response to similar laboratory conditions, as quantified by physiological and subjective sleep measures and neurobehavioral tasks sensitive to sleep loss (e.g., [14-21]). The trait-like susceptibility among individuals accounts for $50-95 \%$ of the variance (depending on the measure) in the severity of neurobehavioral decrements from fatigue due to sleep loss (reviewed in $[22,23])$. The differences among healthy people in neurobehavioral decrements are large (i.e., order of magnitude) and stable (trait-like or phenotypic) over time. In healthy adults, approximately a third have profound performance deficits with even moderate sleep loss (i.e., type 3 response); a third have moderate deficits (i.e., type 2 response), and rather remarkably, a third have few or no performance deficits (i.e., type 1 response), even when sleep loss is severe [22, 23].

Moreover, a number of behavioral markers of susceptibility to neurobehavioral decrements from sleep loss are notably stable and trait-like, across long durations (months to years; Goel, unpublished). That is, those who are resilient to the effects of sleep loss (either total sleep deprivation or chronic sleep restriction), somewhat susceptible, or very susceptible show consistent neurobehavioral responses when reexposed to the same sleep loss conditions months to years later. Prior sleep loss performance is therefore a strong predictor of individual responses to subsequent sleep deprivation exposures, occurring chronically or intermittently, across months and years (Goel, unpublished).

With such significant stability and with such a large amount of susceptibility variance tied up in trait factors, there is a substantial need for objective biomarkers/predictors of these differences. This is particularly true since the decrements are not associated with chronotype or habitual sleep need or various demographic factors (e.g., age, gender, race), there are as yet no validated objective predictors, and the susceptibilities are not accurately predicted or introspected by individuals $[14,16,21]$.

To this end, researchers in the field have published studies on candidate gene polymorphisms and individual differences in neurobehavioral responses to sleep loss (reviewed in [22]). While this work is valuable and informative, it is important to also assess biomarkers which can also dynamically change with environmental stimuli, in order to complement ongoing candidate gene work. Being able to reliably predict via biomarkers who is more likely and who is less likely to experience neurobehavioral decrements from sleep loss would offer substantial benefits through more targeted use of countermeasures and optimizing crew utilization in the armed forces, space flight, and occupational settings.

This review begins with a discussion of transcriptomic research for sleep and circadian rhythms in animal models and humans. It next discusses one epigenomic approach, microRNAs (miRNAs), for sleep and circadian rhythms in various model organisms including humans, and then follows with a discussion of metabolomic methodologies in sleep and circadian rhythm research. The notion that such "omics" methodologies can be used to yield biomarkers for predicting individual differences in neurobehavioral responses to sleep loss in humans is proposed.

\section{What is a Biomarker for Sleep Loss?}

A biomarker is formally defined as a proxy that allows remote and early detection of a biological process (e.g., response to sleep loss) regardless of its mechanistic role in the condition being assessed [24]. Although not necessary, ideally, it will also reflect the biology of the original tissue, thus providing insight into mechanism (response to sleep loss) and serve as a potential therapeutic target. A biomarker is validated when it can be connected and integrated with molecular mechanisms across different levels of biological complexity [24].

\section{Transcriptomics}

Transcriptomics or expression profiling is the study of the complete set of RNA transcripts that are produced by the genome (transcriptome), under specific circumstances or in a specific cell, using high-throughput methods such as microarray analysis or short-read high-throughput sequencing (RNASeq). Comparison of transcriptomes allows the identification of genes that are differentially expressed in distinct cell populations or in response to different conditions. The transcriptome can vary with external environmental conditions.

In recent years, microarray studies have been used to characterize the functions of sleep [25]. These studies have evaluated the genes in specific brain regions whose expression varies between sleep, wakefulness, and sleep deprivation. Such studies have been conducted in rats [26, 27], mice [28, 29], flies [30, 31], and sparrows [32]. Wang et al. [33] have published a comprehensive meta-analysis of transcriptional brain changes in these animal species.

Of interest, the number of differentially expressed transcripts identified varies due to statistical and experimental differences among these studies, including differences in sample size [25]. However, despite these differences, several key pathways commonly are regulated in the brain as a function of sleep-wake behavioral state. Genes involved in synaptic plasticity such as Bdnf, Arc, and Homer 1a [28] are upregulated 
during wakefulness, and cholesterol synthesis [29] is upregulated during sleep. Moreover, across species, sleep deprivation produces upregulation of molecular chaperones [26, 28, 30-33]. Beyond the brain, sleep loss leads to extensive changes in gene expression in other organs, including the liver [28], heart, and lungs [34].

Transcriptomic studies have shown that rhythmic gene expression may be affected by sleep restriction, acute sleep deprivation, and mistimed sleep, whereby the number or amplitude of genes exhibiting circadian rhythmicity is reduced. For example, Maret et al. [28] found that in the presence of a sleep-wake cycle, $\sim 8 \%$ of the brain transcriptome in mice was expressed in a circadian manner, whereas during acute sleep loss, the number of rhythmically expressed transcripts was reduced to $\sim 1.5 \%$, demonstrating a significant acute effect of the sleep-wake cycle on transcription.

In humans, Ackermann et al. [35] found that during acute total sleep deprivation, BMAL1 expression was suppressed, the heat shock gene HSPA1B expression was induced, and the amplitude of the melatonin rhythm increased, whereas other high-amplitude clock gene rhythms (e.g., PER1-3, REVERBa) remained unaffected. These results indicate that the core clock mechanism in peripheral oscillators is compromised during sleep deprivation. Another study found that 1 week of sleep restriction altered gene expression, reduced the amplitude of circadian rhythms in gene expression, and intensified the effects of subsequent acute total sleep loss on gene expression [36•]. The affected genes are involved in chromatin remodeling, gene expression regulation, and immune and stress responses. Thus, remarkably, the effects of sleep history, circadian phase, and acute total sleep loss interact and are reflected in the transcriptome. In another study from this group [37], desynchrony between sleep and circadian phase (i.e., mistimed sleep) led to a reduction of rhythmic transcripts in the human blood transcriptome from $6.4 \%$ to $1.0 \%$, even though the centrally driven circadian rhythm of melatonin was not affected.

Two recent gene expression studies have begun to identify the molecular mechanisms relating to interindividual variation. Pellegrino et al. [38] evaluated the gene expression profiles of subjects who underwent $60 \mathrm{~h}$ of sleep deprivation followed by $12 \mathrm{~h}$ of recovery. They found that expression of multiple genes was downregulated following sleep deprivation and upregulated after recovery compared with sleep deprivation, suggesting an attempt to reestablish internal homeostasis. Notably, the authors detected individual differences in the global expression pattern at baseline and during sleep deprivation, highlighting the fact that not all subjects show similar gene expression during exposure to the same conditions.

In the second study relating to interindividual variation, Arnardottir and colleagues [39•] investigated whether gene expression changes in blood cells induced by sleep loss were different in individuals resistant and sensitive to acute sleep deprivation (as determined by performance on the Psychomotor Vigilance Test (PVT), a behavioral attention test sensitive to sleep loss [40]). The sensitive and resistant subjects had no differences in PVT performance or gene expression patterns under normal sleep-wake conditions, and both groups showed increased PVT lapsing with sleep deprivation, with the magnitude of these lapses significantly greater in the sensitive subjects. However, the authors delved deeper into their data and compared the distribution of cycling gene probes using different $P$ values across $24 \mathrm{~h}$ at baseline and during sleep deprivation in resistant and sensitive subjects for all 52,378 probes (Fig. 1). At baseline, sensitive subjects with a high number of PVT lapses and resistant subjects with a low number of PVT lapses had similar numbers of significantly cycling probe sets. However, during sleep deprivation, resistant subjects had far fewer differentially cycling probe sets than sensitive subjects at any given $P$ value (Fig. 1). Sleep deprivation also led to a reduction in the amplitude of the diurnal rhythm of expression of normally cycling probe sets; this reduction was noticeably higher in behaviorally resistant subjects than sensitive subjects. As noted by Warby and Mongrain [41], this finding suggests that behaviorally resistant subjects responded to extended wakefulness by dampening their oscillating expression patterns. Such a response may be advantageous during unanticipated sleep-wake states, and individuals resistant to sleep deprivation may be able to do this more efficiently than sensitive individuals [41]. By contrast,

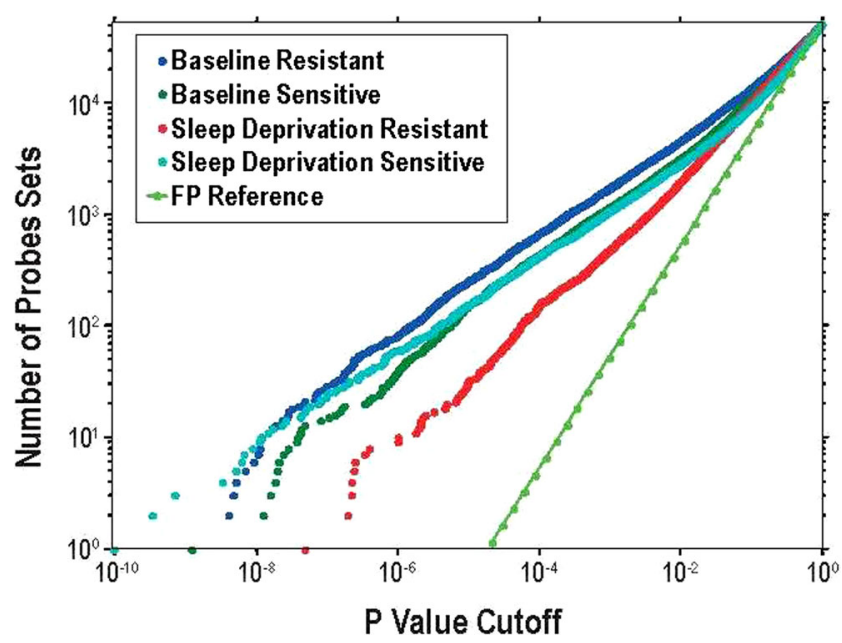

Fig. 1 Decreased number of cycling probe sets in behaviorally resistant subjects during sleep deprivation. The graph shows the cumulative distribution of the number of cycling probe sets across $24 \mathrm{~h}$ ( $y$-axis) below a specified $P$ value ( $x$-axis) during baseline and sleep deprivation. Data are shown for behaviorally sensitive subjects and resistant subjects separately. Although both groups are almost identical at baseline, the resistant subjects show markedly fewer cycling probe sets during sleep deprivation than sensitive subjects. The reference line represents the expected number of false positives (FP) calculated based on the uniform $P$ value distribution that is expected under the null hypothesis. Reprinted with permission from [39•] 
behaviorally sensitive subjects showed transcriptional resistance to the altered conditions; they failed to make the necessary molecular adaptations and continued to express genes in a daily fashion as though they were experiencing a normal sleep-wake cycle [41]. This study provides compelling initial data for the use of transcriptome changes as a means to potentially determine whether a person is sensitive or resistant to sleep deprivation and to manipulate the expression of certain genes as countermeasures for mitigating neurobehavioral vulnerability.

\section{Epigenomics}

Epigenomics involves chemical changes to the genome that affect the manner in which DNA is packaged and expressed without altering its sequence. These can lead to lasting and heritable gene transcription changes but also can be reversible in response to changes in the environment [42]. One important epigenetic mechanism is miRNAs.

\section{microRNAs}

miRNAs are short, single-stranded RNAs (about 22 nucleotides long) processed from $\sim 70$ nucleotide, genomically encoded primary transcripts that potently inhibit gene expression from target messenger RNAs (mRNAs). Although miRNAs mechanism of action is unclear, it involves base pairing between the mRNA 3 '-untranslated region (UTR) and miRNA 5'-end, leading to translational inhibition and/or increased mRNA degradation and/or mRNA sequestration [43]. miRNAs are novel regulators of gene expression that target components of many signal transduction pathways [42]. More than one third of human genes are regulated by miRNAs in diverse aspects of cell physiology [44]. However, the complexity of the miRNA actions is substantial, involving: multiple target mRNAs, some degraded and others stabilized; time-of-day-dependent transcription of many of the target mRNAs; and differential rates of translation of each target mRNA.

Because miRNAs can interfere with mRNA translation, sleep deprivation was predicted to produce concomitant changes of miRNA levels in the brain. In rats, miR-138, let$7 \mathrm{~b}$, and miR-125a were upregulated in the hippocampus and downregulated in the hypothalamus and prefrontal and somatosensory cortices following sleep deprivation [45]. miR132, a mediator of synaptic plasticity, was decreased after sleep loss in both the hippocampus and cortex. Notably, intracerebroventricular application of a miRNA-132 mimetic (preMIR-132) decreased nonrapid-eye-movement sleep duration while simultaneously increasing rapid eye movement sleep duration during the light phase. Further, preMIR-132 decreased electroencephalographic slow-wave activity during non-rapid eye movement sleep, an index of sleep intensity [46]. In another study by this group [47], miR-138, let-7b, and miR-125a inhibitors given intraventricularly differentially altered sleep-wake durations and non-rapid eye movement sleep EEG slow-wave activity. Moreover, sleep deprivation altered four miRNAs (miR-410, miR-212, and miR-29c and miR151) independent of elevated corticosterone levels [48]. In addition, sleep deprivation via fragmentation in mice also resulted in significant miRNA changes in adipose tissue [49]. Thus, many miRNAs, including miR-138, let-7b, and miR-125a, and miR-132, play a clear role in sleep regulation.

In humans, miRNAs have been linked to central nervous system diseases, including neurological disorders [24]. The role of miRNAs in human sleep regulation and sleep disturbances has only begun to be investigated. Holm and colleagues [50 ${ }^{\bullet}$ found that four miRNAs differed in plasma in patients with narcolepsy and other central hypersomnias compared with controls. Levels of miR-30c, let-7f, and miR-26a were higher, whereas the level of miR-130a was lower in type 1 narcolepsy than healthy controls. In addition, the miRNA differences were not specific to type 1 narcolepsy: the levels of the four miRNAs were also altered in patients with type 2 narcolepsy and idiopathic hypersomnia compared with healthy controls. These findings suggest that miRNA alterations may be involved in the pathophysiology of these sleep disorders.

Transcriptional/posttranslational feedback loops have been well characterized in the regulation of circadian rhythms in flies and rodents. Recent research has focused on the role of miRNAs as posttranscriptional regulators of the circadian clock and circadian rhythms. In the SCN of mice, miR-139 and miR-219 modulate the clock entrainment and its period, respectively [51], and in flies, manipulations of miR-279 levels impact rest-activity rhythms [52]. A number of studies provide further support for the involvement of miRNAs in clock timing processes [53-55]. In addition, Nagel et al. [56] identified the miR-192/194 cluster as a potent inhibitor of the Period gene family (Per 1, Per 2, and Per 3) such that exogenous expression of miR-192/194 produced altered circadian rhythms. Genetic disruption of the miRNA pathway by mutating Dicer either in the mouse or in cell lines has also been shown to affect the circadian transcriptome [44, 57].

Thus, multiple miRNA species have been implicated in the molecular clock and output pathways in several model organisms. While the role of miRNAs on the circadian clock in animal models has been extensively studies in recent years, much less is known about the role of miRNAs on the circadian clock in humans.

In a study in shift workers, Shi and colleagues [58] found that long-term night shift work was associated with miR-219 hypermethylation. In a clinical population, Saus et al. [59] found that a polymorphism in pre-miR-182 was associated with late insomnia in major depression. Relative to wild-type 
miR-182, mutant miR-182 (T allele of the rs76481776 polymorphism) triggered a marked reduction in the expression of the circadian gene CLOCK. Additionally, the expression levels of five miRNAs (miRNA-26b, miRNA-1972, miRNA-4485, miRNA-4498, and miRNA-4743) were upregulated in major depressive disorder patients compared with controls [60]. Moreover, $\mathrm{Xu}$ et al. [61] detected a positive association between miRNA-30e ss178077483 and major depression. In bipolar depression patients, Rong et al. [62] found that miR-134 levels were lower compared with controls and higher following medication use. Overall, these data raise the possibility that various miRNAs may be directly associated with the pathophysiology and severity of major depression and bipolar depression; both these disorders show disruptions of circadian rhythms and sleep which may underlie the development of these conditions [63]. These miRNAs should be targeted as biomarkers for predicting differential responses to sleep loss in these clinical populations as well as in healthy subjects without mood disorders.

\section{Metabolomics}

Metabolomics - the global analysis of metabolites (small molecules) - has emerged as a powerful approach used in many disciplines to study how a stimulus causes perturbations in the metabolite profile of a biological sample. Metabolomics has an advantage over other omics techniques, because it directly samples metabolic changes and integrates information from changes at the gene, transcript, and protein levels, as well as at the posttranslational modification level [64•]. Metabolomics can also provide information about genotype and environmental interactions.

Studies have identified circadian influences on the hepatic metabolome in mice $[65,66]$. Plasma metabolites using a mouse model that vary significantly with time of day have crucially also been identified [67]. In humans, initial studies investigated time-of-day variation in the metabolome by comparing the metabolomic profiles of morning versus early evening urine samples $[68,69]$. Although both studies identified significant changes in metabolite levels, comparison of samples collected at only two time points did not provide a comprehensive overview of variation across the 24-h day. Furthermore, these studies did not control for confounds such as sleep, activity, medication, alcohol/caffeine intake, or environmental lighting.

In the past few years, various metabolites have been found to show circadian variations in humans. Remarkably, one study using constant routine and forced desynchrony protocols found that metabolites from blood taken every $2 \mathrm{~h}$, which were used to form a circadian timetable, could subsequently be used to predict internal time within a 3-h interval using only two blood samples [70]. This finding has major implications for the use of personalized medicine and the administration of countermeasures for individual responses to environmental conditions including changes in circadian rhythms and sleep.

A number of studies have collectively identified multiple potential biomarkers of human circadian phase. Dallmann et al. [71] used a constant routine protocol to examine pooled metabolites in saliva and plasma at different times of day, using a limited metabolomic profile (281 metabolites). The authors found that $\sim 15 \%$ of all identified metabolites in plasma and saliva were under circadian control, including fatty acids in plasma and amino acids in saliva. These data indicate a direct effect of the endogenous circadian clock on multiple human metabolic pathways relatively independent of sleep or feeding. Another study assessed changes in metabolites using fixed sleep-wake schedule and dietary regimen for 1 week at home prior to an adaptation night and followed by a 25-h experimental session in the laboratory where the lightdark cycle, sleep-wake, posture, and caloric intake were strictly controlled [72]. A total of 1069 metabolite features were detected, and 203 (19\%) showed significant time-of-day variation, including corticosteroids, bilirubin, amino acids, acylcarnitines, and phospholipids.

A recent study focused on interindividual differences in lipid metabolite profiles. Chua et al. [73•] used targeted lipidomics-based approaches to profile the time course of 263 lipids in blood plasma in healthy men. Across subjects, about $13 \%$ of lipid metabolites showed circadian variation. Rhythmicity spanned all metabolite classes examined, suggesting widespread circadian control of lipid-mediated energy storage, transport, and signaling. As shown in Fig. 2, the intersubject agreement for rhythmic lipids was only about $20 \%$, and the timing of lipid rhythms ranged up to $12 \mathrm{~h}$ apart between individuals. Healthy subjects showed substantial variation in the timing and strength of rhythms across different lipid species (Fig. 2). Robust interindividual differences were also observed for rhythms of blood glucose and insulin, but not for cortisol (Fig. 2). In addition, subjects clustered into different groups based on the strength of rhythmicity for a subset of triglycerides and phosphatidylcholines. The substantial variation in the timing and strength of lipid rhythms across different lipid species indicates that different circadian metabolic phenotypes exist in the general population. The lipids that show such variation may predict individual differences in neurobehavioral performance to sleep deprivation and are worth further examination.

Two recent studies have identified multiple potential metabolomic biomarkers of human sleep and sleep deprivation. Davies et al. [64•] examined metabolic profiling during sleep and acute total sleep deprivation and the characterization of 24-h rhythms under these conditions in healthy males. Subjects remained in controlled laboratory conditions with respect to environmental light, sleep, meals, and posture 
A

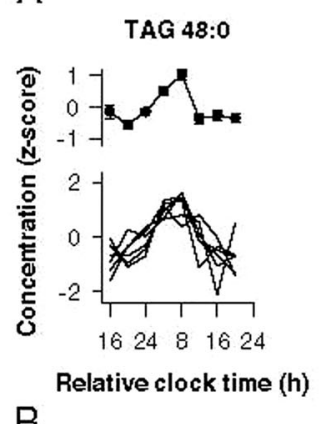

B

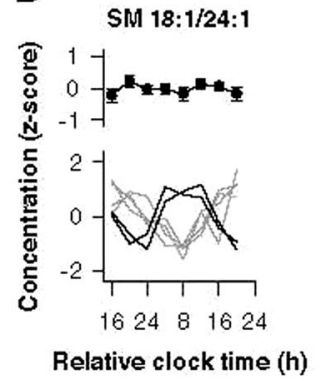

$\mathrm{C}$
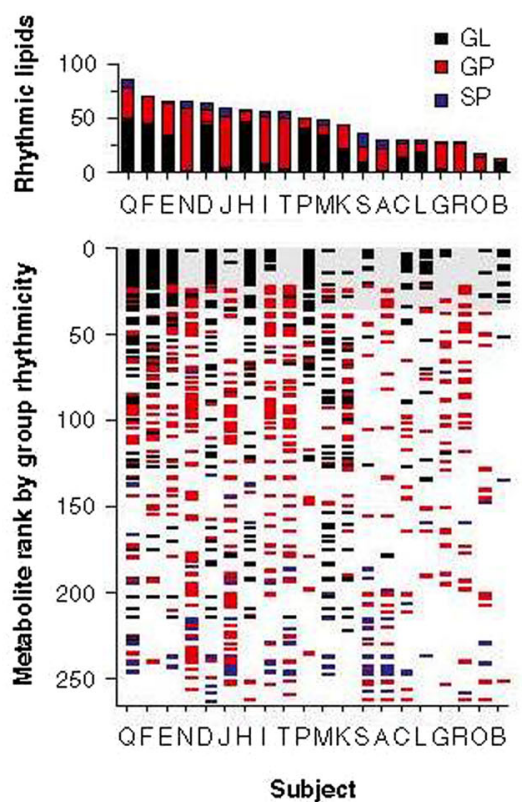

D

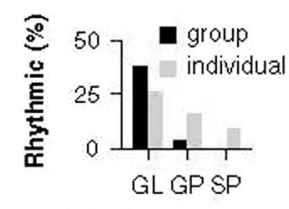

E

Metabolite category

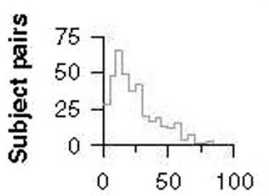

F

Agreement $(\%)$

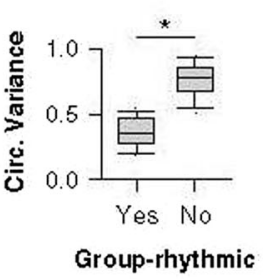

G
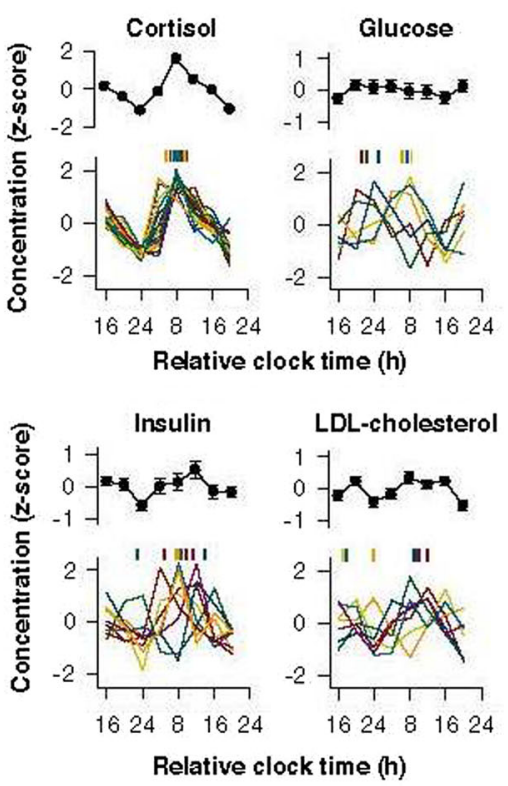

Fig. 2 Interindividual differences in circadian control of lipids. a A representative triglyceride (TAG) is shown that was identified as group rhythmic (top), with rhythmicity driven by six participants with a similar 24-h profile. b A sphingomyelin (SM) species is shown that was not rhythmic based on group-level analysis (top) but was nonetheless rhythmic in six subjects (bottom) with differently timed rhythms (gray and black traces). In the top of $\mathbf{a}$ and $\mathbf{b}$, the mean $\pm \operatorname{SEM}$ is shown $(n=20)$. c Bar graph shows the number of rhythmic metabolites in each subject (labeled $A-T$ ) across different lipid categories (upper). In the scatter plot, metabolites are indicated that were rhythmic on a per-individual basis (lower) (glycerolipids, GL, black; glycerophospholipids, GP, red; sphingolipids, SP, blue). From top to bottom, metabolites are arranged in order of group rhythmicity assessed using the JTK_CYCLE algorithm. Gray box shows the 35 lipid species identified as group rhythmic. d

during a 24-h sleep-wake cycle, followed by $24 \mathrm{~h}$ of wakefulness. Of 171 metabolites quantified, daily rhythms were observed in 109 , with 78 of these maintaining their rhythmicity during $24 \mathrm{~h}$ of wakefulness, albeit 66 with reduced amplitude. During acute total sleep deprivation, 27 metabolites (16\%) including tryptophan, serotonin, taurine, 8 acylcarnitines, 13 glycerophospholipids, and 3 sphingolipids were increased compared with during sleep. In another study, using controlled food intake, and only a limited metabolomic profile (362 metabolites), chronic sleep restriction of eight nights of $5.5 \mathrm{~h}$ of sleep increased 12 amino acids and related metabolites in adults compared with eight nights of $8.5 \mathrm{~h}$ of sleep [74]. Sleep restriction also induced elevations in several fatty acid, bile acid, steroid hormone, and tricarboxylic acid cycle intermediates. In contrast, circulating levels of glucose, some monosaccharides, gluconate, and five-carbon sugar alcohols showed a tendency to decline as a result of sleep restriction [74].

It remains unknown how chronic sleep restriction and subsequent recovery will change metabolomic profiles under ad lib feeding conditions. In addition, translational approaches
Percentage of group rhythmic versus individually rhythmic metabolites is shown across different lipid categories. e Distribution of agreement across circadian lipid profiles is shown for all pairwise comparisons between subjects. $\mathbf{f}$ Box plots show the distribution of circular variance in circadian phase for metabolites in which six or more participants displayed circadian rhythmicity (group rhythmic, 30 lipid species; group arrhythmic, 32 lipid species). Boxes show the interquartile range, and whiskers indicate the 90th percentile of the distribution. $\mathrm{g}$ Black traces show the time course of plasma cortisol, glucose, insulin, and LDL cholesterol averaged across all subjects (mean $\pm \mathrm{SEM}$ ), and colored traces show individual profiles with circadian variation. For individual rhythms, the fitted peak is indicated at the top of each plot by vertical marks of the same color. Reprinted with permission from [73•]

have not yet been undertaken to investigate metabolomic markers of sleep homeostasis in multiple species (e.g., flies, rodents, humans). Moreover, it is unknown whether specific metabolites might predict individual differences in neurobehavioral responses to sleep loss such that increased or decreased levels of certain metabolites could offset the negative performance consequences of sleep loss and be used as an effective countermeasure. Similar to individual differences in neurobehavioral functioning, we have observed individual differences in energy balance measures including weight gain and caloric intake, in response to sleep restriction $[75,76]$. It is plausible that specific changes in the metabolome could also serve as biomarkers for predicting such metabolic differences.

\section{Conclusions}

This review discusses omics methodologies (transcriptomics, epigenomics, and metabolomics) in sleep and circadian rhythm research. Since the molecular mechanisms underlying 
differential vulnerability remain virtually unknown, such methodologies can be used to yield biomarkers for predicting individual differences in neurobehavioral response to sleep loss. There will likely be different biomarkers which predict different behavioral, physiological, and metabolic responses. Thus, a composite of such biomarkers which complement one another is needed. Among other advantages, identification of such markers will provide a viable means to determine those individuals in the general population who may need more habitual sleep or who may need to prevent or mitigate sleep deprivation through lifestyle choices and effective interventions and countermeasures (e.g., caffeine, naps, etc.). If such a set of biomarkers for susceptibility to the neurobehavioral effects of sleep loss is found, it will also be possible to optimize personnel resources and mitigate performance risks across various occupational settings. Indeed, "omics" markers recently were highlighted as of importance for determining individual differences in astronauts and developing countermeasures for astronaut performance and safety in space flight [77].

Acknowledgments Preparation of this manuscript was supported by ONR N00014-11-1-0361, NASA NNX14AN49G, and NIH MH102310.

\section{Compliance with Ethics Guidelines}

Conflict of Interest Namni Goel declares that she has no conflict of interest.

Human and Animal Rights and Informed Consent This article does not contain any studies with human or animal subjects performed by the author.

\section{References}

Papers of particular interest, published recently, have been highlighted as:

- Of importance

1. Van Dongen HPA, Dinges DF. Investigating the interaction between the homeostatic and circadian processes of sleep-wake regulation for the prediction of waking neurobehavioral performance. J Sleep Res. 2003;12:181-7.

2. Goel N, Basner M, Rao H, Dinges DF. Circadian rhythms, sleep deprivation and human performance. Prog Mol Biol Transl Sci. 2013;119:155-90.

3. Achermann P, Dijk DJ, Brunner DP, Borbély AA. A model of human sleep homeostasis based on EEG slow-wave activity; quantitative comparison of data and simulations. Brain Res Bull. 1993;31:97-113.

4. Borbély AA. A two process model of sleep regulation. Hum Neurobiol. 1982;1:195-204.

5. Mallis MM, Mejdal S, Nguyen TT, Dinges DF. Summary of the key features of seven biomathematical models of human fatigue and performance. Aviat Space Environ Med. 2004;75:A4-14.
6. Daan S, Beersma DGM, Borbély AA. Timing of human sleep: recovery process gated by a circadian pacemaker. Am J Physiol. 1984;246:R161-78.

7. Achermann P, Borbély AA. Simulation of daytime vigilance by the additive interaction of a homeostatic and a circadian process. Biol Cybern. 1994;71:115-21.

8. Khalsa SBS, Jewett ME, Duffy JF, Czeisler CA. The timing of the human circadian clock is accurately represented by the core body temperature rhythm following phase shifts to a three-cycle light stimulus near the critical zone. J Biol Rhythm. 2000;15:524-30.

9. Edgar DM, Dement WC, Fuller CA. Effect of SCN lesions on sleep in squirrel monkeys: evidence for opponent processes in sleepwake regulation. J Neurosci. 1993;13:1065-79.

10. Doran SM, Van Dongen HPA, Dinges DF. Sustained attention performance during sleep deprivation: evidence of state instability. Arch Ital Biol. 2001;139:253-67.

11. Banks S, Dinges DF. Behavioral and physiological consequences of sleep restriction in humans. J Clin Sleep Med. 2007;3:519-28.

12. Goel N, Rao H, Durmer JS, Dinges DF. Neurocognitive consequences of sleep deprivation. Semin Neurol. 2009;29:320-39.

13. Lim J, Dinges DF. A meta-analysis of the impact of short-term sleep deprivation on cognitive variables. Psychol Bull. 2010;136:375-89.

14. Van Dongen HPA, Baynard MD, Maislin G, Dinges DF. Systematic interindividual differences in neurobehavioral impairment from sleep loss: evidence of trait-like differential vulnerability. Sleep. 2004;27:423-33.

15. Van Dongen HPA, Maislin G, Dinges DF. Dealing with interindividual differences in the temporal dynamics of fatigue and performance: importance and techniques. Aviat Space Environ Med. 2004;75:A147-54.

16. Van Dongen HPA, Maislin G, Mullington JM, Dinges DF. The cumulative cost of additional wakefulness: dose-response effects on neurobehavioral functions and sleep physiology from chronic sleep restriction and total sleep deprivation. Sleep. 2003;26:117-26.

17. Goel N, Banks S, Mignot E, Dinges DF. PER3 polymorphism predicts cumulative sleep homeostatic but not neurobehavioral changes to chronic partial sleep deprivation. PLoS One. 2009;4: e5874.

18. Goel N, Banks S, Mignot E, Dinges DF. $D Q B 1 * 0602$ predicts interindividual differences in physiologic sleep, sleepiness and fatigue. Neurology. 2010;75:1509-19.

19. Goel N, Banks S, Lin L, Mignot E, Dinges DF. Catechol-Omethyltransferase Val158Met polymorphism associates with individual differences in sleep physiologic responses to chronic sleep loss. PLoS One. 2011;6:e29283.

20. Bliese PD, Wesensten NJ, Balkin TJ. Age and individual variability in performance during sleep restriction. J Sleep Res. 2006;15:37685.

21. Van Dongen HPA, Dijkman MV, Maislin G, Dinges DF. Phenotypic aspect of vigilance decrement during sleep deprivation. Physiologist. 1999;42:A-5.

22. Goel N. Genetics of sleep timing, duration and homeostasis in humans. Sleep Med Clin. 2011;6:171-82.

23. Goel N, Dinges DF. Predicting risk in space: genetic markers for differential vulnerability to sleep restriction. Acta Astronaut. 2012;77:207-13.

24. Rao P, Benito E, Fischer A. MicroRNAs as biomarkers for CNS disease. Front Mol Neurosci. 2013;6:39.

25. Mackiewicz M, Zimmerman JE, Shockley KR, Churchill GA, Pack AI. What are microarrays teaching us about sleep? Trends Mol Med. 2009;15:79-87.

26. Cirelli C, Gutierrez CM, Tononi G. Extensive and divergent effects of sleep and wakefulness on brain gene expression. Neuron. 2004;41:35-43.

27. Terao A, Wisor JP, Peyron C, Apte-Deshpande A, Wurts SW, Edgar $\mathrm{DM}$, et al. Gene expression in the rat brain during sleep deprivation 
and recovery sleep: an Affymetrix GeneChip study. Neuroscience. 2006;137:593-605.

28. Maret S, Dorsaz S, Gurcel L, Pradervand S, Petit B, Pfister C, et al. Homerla is a core brain molecular correlate of sleep loss. Proc Natl Acad Sci U S A. 2007;104:20090-5.

29. Mackiewicz M, Shockley KR, Romer MA, Galante RJ, Zimmerman JE, Naidoo N, et al. Macromolecule biosynthesis: a key function of sleep. Physiol Genomics. 2007;31:441-57.

30. Cirelli C, LaVaute TM, Tononi G. Sleep and wakefulness modulate gene expression in Drosophila. J Neurochem. 2005;94:1411-9.

31. Zimmerman JE, Rizzo W, Shockley KR, Raizen DM, Naidoo N, Mackiewicz M, et al. Multiple mechanisms limit the duration of wakefulness in Drosophila brain. Physiol Genomics. 2006;27:33750 .

32. Jones S, Pfister-Genskow M, Benca RM, Cirelli C. Molecular correlates of sleep and wakefulness in the brain of the whitecrowned sparrow. J Neurochem. 2008;105:46-62.

33. Wang H, Liu Y, Briesemann M, Yan J. Computational analysis of gene regulation in animal sleep deprivation. Physiol Genomics. 2010;42:427-36.

34. Anafi RC, Pellegrino R, Shockley KR, Romer M, Tufik S, Pack AI. Sleep is not just for the brain: transcriptional responses to sleep in peripheral tissues. BMC Genomics. 2013;14:362.

35. Ackermann K, Plomp R, Lao O, Middleton B, Revell VL, Skene DJ, et al. Effect of sleep deprivation on rhythms of clock gene expression and melatonin in humans. Chronobiol Int. 2013;30:9019.

36. Möller-Levet CS, Archer SN, Bucca G, Laing EE, Slak A, Kabiljo $\mathrm{R}$, et al. Effects of insufficient sleep on circadian rhythmicity and expression amplitude of the human blood transcriptome. Proc Natl Acad Sci U S A. 2013;110:E1132-41. This study demonstrates that one week of sleep restriction alters gene expression, reduces the amplitude of circadian rhythms in gene expression, and intensifies the effects of subsequent acute total sleep loss on gene expression. The affected genes are involved in chromatin remodeling, gene expression regulation and immune and stress responses. The study highlights the molecular interrelationships between sleep homeostasis and circadian rhythmicity.

37. Archer SN, Laing EE, Möller-Levet CS, van der Veen DR, Bucca G, Lazar AS, et al. Mistimed sleep disrupts circadian regulation of the human transcriptome. Proc Natl Acad Sci U S A. 2014;111: E682-91.

38. Pellegrino R, Sunaga DY, Guindalini C, Martins RC, Mazzotti DR, Wei Z, et al. Whole blood genome-wide gene expression profile in males after prolonged wakefulness and sleep recovery. Physiol Genomics. 2012;44:1003-12.

39. Arnardottir ES, Nikonova EV, Shockley KR, Podtelezhnikov AA, Anafi RC, Tanis KQ, et al. Blood-gene expression reveals reduced circadian rhythmicity in individuals resistant to sleep deprivation. Sleep. 2014;37:1589-600. This paper examines whether gene expression changes in blood cells induced by sleep loss are different in individuals resistant and sensitive to acute sleep deprivation. It reports reduced circadian rhythmicity in individuals who are resistant to sleep deprivation, as determined by the Psychomotor Vigilance Test.

40. Lim J, Dinges DF. Sleep deprivation and vigilant attention. Ann N Y Acad Sci. 2008;1129:305-22.

41. Warby SC, Mongrain V. Resisting sleep deprivation by breaking the link between sleep and circadian rhythms. Sleep. 2014;37:1581-2.

42. Stankiewicz AM, Swiergiel AH, Lisowski P. Epigenetics of stress adaptations in the brain. Brain Res Bull. 2013;98:76-92.

43. Nilsen TW. Mechanisms of microRNA-mediated gene regulation in animal cells. Trends Genet. 2007;23:243-9.

44. Chen R, D'Alessandro M, Lee C. miRNAs are required for generating a time delay critical for the circadian oscillator. Curr Biol. 2013;23:1959-68.
45. Davis CJ, Bohnet SG, Meyerson JM, Krueger JM. Sleep loss changes microRNA levels in the brain: a possible mechanism for state-dependent translational regulation. Neurosci Lett. 2007;422: 68-73.

46. Davis CJ, Clinton JM, Taishi P, Bohnet SG, Honn KA, Krueger JM. MicroRNA 132 alters sleep and varies with time in brain. J Appl Physiol. 2011;111:665-72.

47. Davis CJ, Clinton JM, Krueger JM. MicroRNA 138, let-7b, and 125a inhibitors differentially alter sleep and EEG delta-wave activity in rats. J Appl Physiol. 2012;113:1756-62.

48. Mongrain V, Hernandez SA, Pradervand S, Dorsaz S, Curie T, Hagiwara G, et al. Separating the contribution of glucocorticoids and wakefulness to the molecular and electrophysiological correlates of sleep homeostasis. Sleep. 2010;33:1147-57.

49. Gharib SA, Khalyfa A, Abdelkarim A, Bhushan B, Gozal D. Integrative miRNA-mRNA profiling of adipose tissue unravels transcriptional circuits induced by sleep fragmentation. PLoS One. 2012;7:e37669.

50. Holm A, Bang-Berthelsen CH, Knudsen S, Kornum BR, Modvig S, Jennum $P$, et al. miRNA profiles in plasma from patients with sleep disorders reveal dysregulation of miRNAs in narcolepsy and other central hypersomnias. Sleep. 2014;37:1525-33. This study is the first to investigate the role of miRNAs in sleep disorders. It found that 4 miRNAs differed in patients with narcolepsy and other central hypersomnias compared with controls, suggesting that miRNA alterations may be involved in the pathophysiology of these sleep disorders.

51. Cheng HY, Papp JW, Varlamova O, Dziema H, Russell B, Curfman JP, et al. microRNA modulation of circadian-clock period and entrainment. Neuron. 2007;54:813-29.

52. Luo W, Sehgal A. Regulation of circadian behavioral output via a MicroRNA-JAK/STAT circuit. Cell. 2012;148:765-79.

53. Yang M, Lee JE, Padgett RW, Edery I. Circadian regulation of a limited set of conserved microRNAs in Drosophila. BMC Genomics. 2008;9:83.

54. Gatfield D, Le Martelot G, Vejnar CE, Gerlach D, Schaad O, Fleury-Olela F, et al. Integration of microRNA miR-122 in hepatic circadian gene expression. Genes Dev. 2009;23:1313-26.

55. Kadener S, Menet JS, Sugino K, Horwich MD, Weissbein U, Nawathean P, et al. A role for microRNAs in the Drosophila circadian clock. Genes Dev. 2009;23:2179-91.

56. Nagel R, Clijsters L, Agami R. The miRNA-192/194 cluster regulates the period gene family and the circadian clock. FEBS J. 2009;276:5447-55.

57. Du NH, Arpat AB, De Matos M, Gatfield D. MicroRNAs shape circadian hepatic gene expression on a transcriptome-wide scale. eLife. 2014;3:e02510.

58. Shi F, Chen X, Fu A, Hansen J, Stevens R, Tjonneland A, et al Aberrant DNA methylation of miR-219 promoter in long-term night shiftworkers. Environ Mol Mutagen. 2013;54:406-13.

59. Saus E, Soria V, Escaramís G, Vivarelli F, Crespo JM, Kagerbauer $\mathrm{B}$, et al. Genetic variants and abnormal processing of pre-miR-182, a circadian clock modulator, in major depression patients with late insomnia. Hum Mol Genet. 2010;19:4017-25.

60. Fan HM, Sun XY, Guo W, Zhong AF, Niu W, Zhao L, et al. Differential expression of microRNA in peripheral blood mononuclear cells as specific biomarker for major depressive disorder patients. J Psychiatr Res. 2014;59:45-52.

61. Xu Y, Liu H, Li F, Sun N, Ren Y, Liu Z, et al. A polymorphism in the microRNA-30e precursor associated with major depressive disorder risk and P300 waveform. J Affect Disord. 2010;127: 332-6.

62. Rong H, Liu TB, Yang KJ, Yang HC, Wu DH, Liao CP, et al. MicroRNA-134 plasma levels before and after treatment for bipolar mania. J Psychiatr Res. 2011;45:92-5. 
63. Mendlewicz J. Disruption of the circadian timing systems: molecular mechanisms in mood disorders. CNS Drugs. 2009;23:15-26.

64. Davies SK, Ang JE, Revell VL, Holmes B, Mann A, Robertson FP, et al. Effect of sleep deprivation on the human metabolome. Proc Natl Acad Sci U S A. 2014;111:10761-6. This paper assesses metabolic profiles during sleep and acute total sleep deprivation and the characterization of 24-hour rhythms under these conditions in healthy males.

65. Eckel-Mahan KL, Patel VR, Mohney RP, Vignola KS, Baldi $\mathrm{P}$, Sassone-Corsi P. Coordination of the transcriptome and metabolome by the circadian clock. Proc Natl Acad Sci U S A. 2012;109:5541-6.

66. Fustin JM, Doi M, Yamada H, Komatsu R, Shimba S, Okamura H. Rhythmic nucleotide synthesis in the liver: temporal segregation of metabolites. Cell Rep. 2012;1:341-9.

67. Minami Y, Kasukawa T, Kakazu Y, Iigo M, Sugimoto M, Ikeda S, et al. Measurement of internal body time by blood metabolomics. Proc Natl Acad Sci U S A. 2009;106:9890-5.

68. Slupsky CM, Rankin KN, Wagner J, Fu H, Chang D, Weljie AM, et al. Investigations of the effects of gender, diurnal variation, and age in human urinary metabolomic profiles. Anal Chem. 2007;79: 6995-7004.

69. Walsh MC, Brennan L, Malthouse JP, Roche HM, Gibney MJ. Effect of acute dietary standardization on the urinary, plasma, and salivary metabolomic profiles of healthy humans. Am J Clin Nutr. 2006;84:531-9.

70. Kasukawa T, Sugimoto M, Hida A, Minami Y, Mori M, Honma S, et al. Human blood metabolite timetable indicates internal body time. Proc Natl Acad Sci U S A. 2012;109:15036-41.
71. Dallmann R, Viola AU, Tarokh L, Cajochen C, Brown SA. The human circadian metabolome. Proc Natl Acad Sci U S A. 2012;109:2625-9.

72. Ang JE, Revell V, Mann A, Mäntele S, Otway DT, Johnston JD, et al. Identification of human plasma metabolites exhibiting timeof-day variation using an untargeted liquid chromatography-mass spectrometry metabolomic approach. Chronobiol Int. 2012;29: 868-81.

73. Chua EC, Shui G, Lee IT, Lau P, Tan LC, Yeo SC, et al. Extensive diversity in circadian regulation of plasma lipids and evidence for different circadian metabolic phenotypes in humans. Proc Natl Acad Sci U S A. 2013;110:14468-73. This paper used targeted lipidomics-based approaches to profile the time course of 263 lipids in blood plasma in healthy men. The authors report substantial variation in the timing and strength of lipid rhythms across different lipid species, indicating the presence of different circadian metabolic phenotypes in the general population.

74. Bell LN, Kilkus JM, Booth 3rd JN, Bromley LE, Imperial JG, Penev PD. Effects of sleep restriction on the human plasma metabolome. Physiol Behav. 2013;22:25-31.

75. Spaeth AM, Dinges DF, Goel N. Effects of sleep restriction on weight gain, caloric intake and meal timing in healthy adults. Sleep. 2013;36:981-90.

76. Spaeth AM, Dinges DF, Goel N. Sex and race differences in caloric intake during sleep restriction in healthy adults. Am J Clin Nutr. 2014;100:559-66.

77. Schmidt MA, Goodwin TJ. Personalized medicine in human space flight: using Omics based analyses to develop individualized countermeasures that enhance astronaut safety and performance. Metabolomics. 2013;9:1134-56. 